\title{
Studies on the Anisotropic Properties of $\mathrm{MgB}_{2}$
}

\author{
O. F. de Lima \\ Instituto de Física Gleb Wataghin, \\ Universidade Estadual de Campinas - UNICAMP \\ 13083-970 Campinas, SP, Brasil
}

Received on 28 February, 2002

\begin{abstract}
This paper presents a review on reported anisotropic properties of $\mathrm{MgB}_{2}$. The first direct measurement of an anisotropic superconducting property in $\mathrm{MgB}_{2}$ was achieved for the bulk nucleation field $H_{c 2}$, in samples of aligned crystallites. A ratio $H_{c 2}^{a b} / H_{c 2}^{c} \approx 1.7$ was found between the critical field parallel to the $a b$ plane and parallel to the $c$ axis direction. Further, detailed study of the $H_{c 2}$ angular dependence confirmed its bulk origin, in contrast with what would be expected for the surface nucleation field $H_{c 3}$. A Fermi velocity anisotropy was evaluated to be $V_{F}^{a b} \approx 1.6 V_{F}^{c}$, assuming an isotropic order parameter. For an anisotropic s-wave pairing symmetry it has been estimated that $V_{F}^{a b} \geq 2.5 V_{F}^{c}$. Different $H_{c 2}$ anisotropy has been found by different authors, using different samples, measured in varied temperature ranges. Other reported anisotropic properties of $\mathrm{MgB}_{2}$ in the superconducting state are the field penetration depth $\lambda$, coherence length $\xi$, and energy gap $\Delta$; in the normal state are the magnetoresistance, compressibility, and thermal expansion. So far, most of the reported results have been obtained using aligned crystallites, c-axis oriented thin films and sub-millimeter crystals.
\end{abstract}

\section{Introduction}

The discovery of superconductivity at $39 \mathrm{~K}$ in Magnesium Diboride $\left(\mathrm{MgB}_{2}\right)$ [1] has brought new excitement to the area of basic and applied research on superconducting materials.

Several studies have already pointed out that $\mathrm{MgB}_{2}$ has a good potential for applications [2-5] in view of the relatively high values of critical current density, $J_{c}$, and the successful preparation of wires and films. However, intense magnetic relaxation effects, associated with thermally activated flux creep and flux jumps, have been found to limit the $J_{c}$ values at high magnetic fields [6]. This means that effective pinning centers have to be added [7] into the material microstructure, in order to halt dissipative flux movements.

The observation of an isotope effect [8,9], a BCStype energy gap [10], as well as band structure studies [11-13] suggest the occurrence of a phonon-mediated superconductivity in $\mathrm{MgB}_{2}$. Many other experimental [14-18] and theoretical [19, 20] works have pointed also to the relevance of a phonon-mediated interaction, in the framework of the BCS theory. However, the possibility of hole superconductivity, associated with the nearly filled boron planar $\mathrm{p}_{x, y}$ orbitals, has also been suggested [21]. Questions have been raised about the relevant phonon modes, as well as about the gap energy and Fermi surface anisotropies. A considerable amount of spectroscopic and thermal data has shown a broad range of values, between 2.5 and 5.0, for the gap ratio $2 \Delta_{0} / k T_{c}$, where $\Delta_{0}$ is the gap energy at $T$ $=0$ and $k$ is the Boltzmann constant. As a comparison, the BCS theory in the weak coupling limit predicts [22] $2 \Delta_{0} / k T_{c} \approx 3.5$, for an isotropic gap energy. In an effort to interpret this puzzling situation, multiple gap models [19] as well as a general model of anisotropic s-wave order parameter $[18,20]$ have been proposed.

The strongly anisotropic crystalline structure of $\mathrm{MgB}_{2}$ has been known for a long time. It seemed therefore reasonable when specific heat studies done in polycrystalline samples [15] as well as band structure calculations [11], pointed to the possible anisotropic nature of the electronic and magnetic properties of $\mathrm{MgB}_{2}$. The first direct measurement of an anisotropic superconducting property was achieved for the bulk nucleation field $H_{c 2}$, in samples of aligned $\mathrm{MgB}_{2}$ crystallites [23]. It was found a ratio $H_{c 2}^{a b} / H_{c 2}^{c} \approx 1.7$, between the critical field parallel to the $a b$ plane and parallel to the $c$ axis direction. The anisotropic behavior of some normal state properties, like compressibility [24, 25] and magnetoresistance [26], have also been reported.

Following, a brief review of some aspects regarding sample preparation will be presented in Section II. In Section III, a detailed analysis of the bulk nucleation field (or upper critical field) anisotropy will be described. In Section IV other experimentally assessed 
anisotropic properties of $\mathrm{MgB}_{2}$ will be briefly reviewed. Finally, in Section V, a conclusion will be presented. For a more complete review on the superconducting properties of $\mathrm{MgB}_{2}$ see Ref. [27].

\section{Sample preparation}

The compound $\mathrm{MgB}_{2}$ is known to occur in equilibrium with an excess of $\mathrm{Mg}$, for temperatures above $650{ }^{\circ} \mathrm{C}[28]$. By 1953 its $\mathrm{AlB}_{2}$-type crystal structure was determined, using $\mathrm{X}$-ray diffraction studies $[29,30]$. This layered structure belongs to the space group P $6 / \mathrm{mmm}$, and consists of alternating triangular layers of $\mathrm{Mg}$ atoms and graphite-like hexagonal layers of $\mathrm{B}$ atoms. The unit cell lattice parameters typically reported $[29,30,31,32]$ are around $a=3.085 \AA$ and $c$ $=3.521 \AA$.

A common route to prepare $\mathrm{MgB}_{2}$ polycrystalline samples starts by mixing the pure elements, $\mathrm{Mg}$ and $\mathrm{B}$, in the atomic ratio $\mathrm{B}: \mathrm{Mg}=2: 1$. Because of the relatively high vapour pressure of $\mathrm{Mg}$ it is advised to react the mixture sealed in an evacuated tube or container of an appropriate material (e.g. Ta, Nb, Fe, Mo, BN). In order to avoid oxidation of the tube/container external surface, especially in cases when open-air furnaces are used, the sealed tube/container is sealed inside a quartz ampoule, in a residual atmosphere of argon gas. Typically, a reaction time around 2 hours at temperatures between $700{ }^{\circ} \mathrm{C}$ and $1000{ }^{\circ} \mathrm{C}$ have been used, followed by cooling to room temperature. However, to obtain small single crystals, a higher reaction temperature between $1200-1400{ }^{\circ} \mathrm{C}$ and addition of $\mathrm{Mg}$ in excess to provide an internal vapour pressure above 1 bar, was reported in 1973 [31]. This is essentially the same route employed recently by different groups, to obtain $\mathrm{MgB}_{2}$ crystallites having sub-millimeter sizes [23,33]. A different approach consists of reacting the quasiternary $\mathrm{Mg}-\mathrm{MgB}_{2}-\mathrm{BN}$ system, at pressures around 50 bar and temperatures ranging between $1400-1700{ }^{\circ} \mathrm{C}$, for time periods between 5 to 60 minutes. In this case $\mathrm{MgB}_{2}$ crystals reaching linear dimensions up to $0.7 \mathrm{~mm}$ has been reported [32].

$\mathrm{MgB}_{2}$ thin films have also been grown successfully, using pulsed laser deposition $[4,34]$ as well as by electron beam evaporation of $\mathrm{B}$ and $\mathrm{Mg}$ [35]. In some cases textured thin films were obtained, consisting of grains that have their crystallographic $c$ axis aligned perpendicularly to the substrate plane. An intriguing feature of thin films is that their $T_{c}$ ranges typically between $25-37 \mathrm{~K}$, always below the bulk value of $39 \mathrm{~K}$.

\section{II.1. Aligned $\mathrm{MgB}_{2}$ crystallites}

While polycrystalline $\mathrm{MgB}_{2}$ is very easy to grow and is a readily available reagent, good-sized single crystals of this material, with linear dimensions above 0.7 $\mathrm{mm}$ have not yet been reported, and their development promises to keep being a real challenge. However, by the middle of February 2001, in the early days of intense activities on the new $\mathrm{MgB}_{2}$ superconductor, samples of aligned $\mathrm{MgB}_{2}$ crystallites were prepared at UNICAMP [23]. Firstly, a weakly sintered sample of $\mathrm{MgB}_{2}$ was synthesised, starting from a stoichiometric mixture of 99.5 at\% pure B and 99.8 at\% pure $\mathrm{Mg}$, both in chips form (Johnson Matthey Electronics). The loose mixture was sealed in a Ta tube under Ar atmosphere, which was then encapsulated in a quartz ampoule and put into the furnace. The compound formation was processed by initially holding the furnace temperature at $1200^{\circ} \mathrm{C}$ for 1 hour, followed by a decrease to $700{ }^{\circ} \mathrm{C}\left(10^{\circ} \mathrm{C} / \mathrm{h}\right)$, then to $600{ }^{\circ} \mathrm{C}\left(2{ }^{\circ} \mathrm{C} / \mathrm{h}\right)$, and finally to room temperature at a rate of $100^{\circ} \mathrm{C} / \mathrm{h}$. The weakly sintered product was easily crushed and milled by employing mortar and pestle. Using a stereomicroscope one could observe at this stage a very uniform powder, consisting mainly of shiny crystallites with aspect ratios ranging from 2 to 5 and linear dimensions going up to $50 \mu \mathrm{m}$. The powder was then sieved into a range of particle sizes between $5-20 \mu \mathrm{m}$, which allowed the crystallites fraction to be maximised to almost $100 \%$. Small amounts of the powder were then patiently spread on both sides of a small piece of paper, producing an almost perfect alignment of the crystallites laid down on top of the flat surface, as shown in the SEM picture of Fig. 1. The success of this method relies on the crystallites platelike shape, which is indeed a macroscopic manifestation of its anisotropic crystalline structure. Fig. 2 shows an $\mathrm{X}$-ray diffraction pattern $(\theta-2 \theta$ scan $)$ measured on one of the aligned sample, displaying only the (001) and (002) reflections, coming from the $\mathrm{MgB}_{2}$ phase. A lattice parameter $\mathrm{c}=3.518 \pm 0.008 \AA$ was evaluated from these two peak positions. The two small impurity peaks marked with asterisks were indexed as $\mathrm{SiO}_{2}$. The inset of Fig. 2 shows a rocking curve ( $\omega$ scan) for the (002) peak that reveals an angular spread (Full Width at Half Maximum) around 4.6 degrees, associated with a small misalignment of the crystallites $c$ axis.

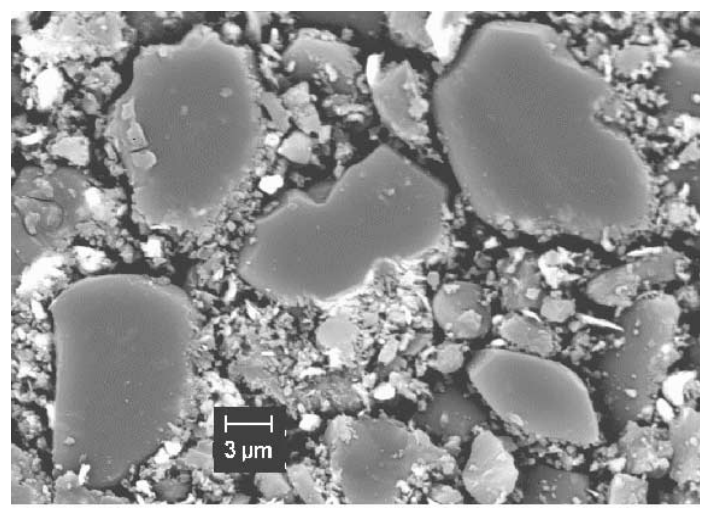

Figure 1. Scanning Electron Microscopy (SEM) picture showing the well-aligned crystallites and intercrystallite material. 


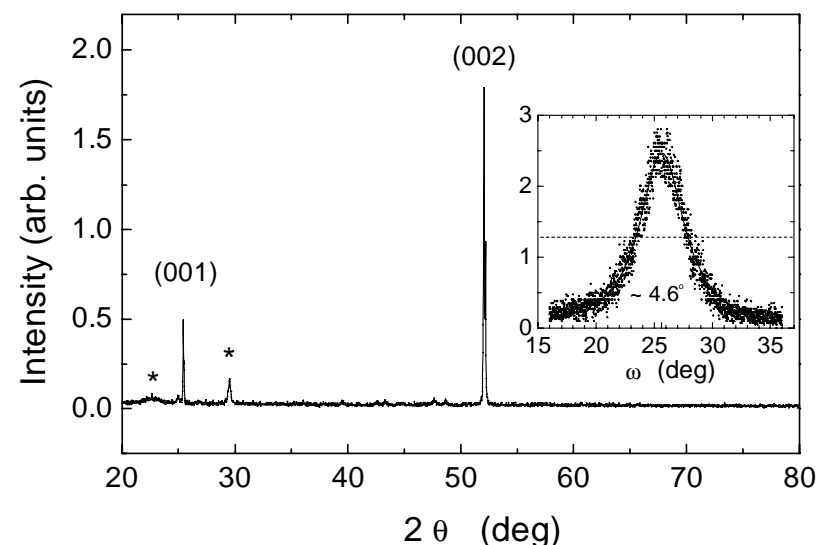

Figure 2. X-ray diffraction pattern showing only the (001) and (002) peaks of $\mathrm{MgB}_{2}$, plus two spurious peaks indexed as $\mathrm{SiO}_{2}$. Inset: rocking curve $(\omega-$ scan $)$ for the $(002)$ peak, showing an angular spread of about 4.6 degrees along the crystallites $c$ axis.

Electron microprobe analysis done on four different areas between the $\mathrm{MgB}_{2}$ crystallites, revealed the following average concentration (in at\%) of elements: $\mathrm{O}$ (62.9), C (22.2), Ca (9.48), Si (1.48), Mg (1.44), Al (1.37), K (0.09), Fe (0.50), Cr (0.21), Ni (0.09). The first eight elements in this list were found also in the composition analysis made on the same type of paper used (Canson, ref. 4567-114). Microprobe analysis done also on the initial $\mathrm{Mg}$ and $\mathrm{B}$ revealed a few small precipitates, smaller than $10 \mu \mathrm{m}$ and containing up to 8 at\% Fe, only in the Mg chips. This confirms the expectation of Fe being a common impurity [36] in commercial $\mathrm{Mg}$, and sets a general concern on its possible effects, although recent reports [5,37] have suggested no negative effects to the superconductivity of $\mathrm{MgB}_{2}$ from Fe additions. The average composition found on top of several crystallites, normalised to the whole $\mathrm{MgB}_{2}$ formula unit, was: $\mathrm{Mg}(30.80), \mathrm{O}$ (2.20), Ca (0.17), Si (0.07), Fe (0.06). Although Boron contributes with a fraction of 66.6 at \% it does not show-up in the microprobe analysis because it is too light. The contaminants found on top of the crystallites most possibly came from a surface contamination caused by the alignment technique, which required vigorous rubbing on top of the powder, using a steel tweezers tip to spread the crystallites uniformly. This is corroborated by a further analysis done on top of several as-grown crystallites, which detected only $\mathrm{Mg}$ and a small amount of $\mathrm{O}$ (possibly from $\mathrm{MgO}$ ). This result is consistent with the very small solid solubility limit of about 0.004 at\% Fe in $\mathrm{Mg}$, which is known to occur [38] at the solidification temperature of $650{ }^{\circ} \mathrm{C}$. The inter-crystallite type of rubbish shown in Fig. 1 is attributed mainly to the paper abrasion, which produces a varied distribution of irregular grains of paper fragments. In order to characterise the superconducting and magnetic properties of the aligned crystallites several samples were mounted, consisting of a pile of five squares of $3 \times 3 \mathrm{~mm}^{2}$, cut from the crystallite-painted paper and glued with Araldite resin. Each one of these samples contains a number of crystallites estimated to be around $6.5 \times 10^{5}$, ending up with an effective volume of $0.065 \mathrm{~mm}^{3}$. This value is reasonably close to $0.060 \mathrm{~mm}^{3}$ that was evaluated from the expected slope $M / H=-1 / 4 \pi$, when the magnetization $M$ is measured at a very low field $H$.

\section{III $H_{c 2}$ anisotropy}

$H_{c 2}$ anisotropy is one of the macroscopic manifestations of an anisotropic gap energy or an anisotropic Fermi surface, as well as a possible combination of both effects $[39,40]$. Therefore, its study is of paramount importance to help understanding the basic mechanisms involved in the pairing interaction. Samples of aligned $\mathrm{MgB}_{2}$ crystallites, as described above, have been employed in detailed studies of the $H_{c 2}$ angular dependence, providing a clear identification of its anisotropy factor and its bulk origin [23, 41].

Figure 3 shows the anisotropic signature of the $H_{c 2}$ line in the field interval $0 \leq H \leq 40 \mathrm{kOe}$. The experimental points were taken from the transition onset of the real component $\left(\chi^{\prime}\right)$ of AC susceptibility, measured using a PPMS-9T machine (Quantum Design), with an excitation field of amplitude $1 \mathrm{Oe}$ and frequency $5 \mathrm{kHz}$. The inset shows an enlarged view of the $\chi^{\prime}(T)$ curves for $H / / a b$ (open symbols) and $H / / c$ (solid symbols), the field orientation parallel to the $a b$ plane and parallel to the $c$ axis, respectively. The $\chi^{\prime}(T)$ as well as the $M(T)$ (inset of Fig. 4) measurements, for $H=$ 10 Oe, show sharp transitions at the same critical temperature $T_{c} \approx 39 \mathrm{~K}$. Typically, some of the published data on the tempera ture dependence of $H_{c 2}[42,43]$ agree with the result displayed in Fig. 3 for $H_{c 2} / /$ $a b$. As an example, the data from Ref.[43] is plotted in Fig. 3 as stars. This mean that in polycrystalline samples the transitions are broadened, showing the onset at the highest temperature that corresponds to the highest critical field available, which is $H_{c 2} / / a b$.

The ratio $H_{c 2}^{a b} / H_{c 2}^{c}$, between the upper critical field when $H$ is applied parallel to the $a b$ plane and when it is along the $c$ direction, was evaluated at different temperatures, producing a value around 1.7. From [22] $H_{c 2}^{a b} / H_{c 2}^{c}=\xi_{a b} / \xi_{c}, H_{c 2}^{a b}(T)=\Phi_{0} /\left(2 \pi \xi_{a b} \xi_{c}\right) H_{c 2}^{c}(T)=$ $\Phi_{0} /\left(2 \pi \xi_{a b}^{2}\right)$, and using the Ginzburg-Landau (G-L) mean field expression for the coherence length, $\xi(T)=$ $\xi_{0}\left(1-T / T_{c}\right)^{-0.5}$, one finds $\xi_{0, a b} \approx 70 \AA$ and $\xi_{0, c} \approx 40 \AA$, the coherence length at $T=0$ in the $a b$ planes and along the $c$ axis, respectively. The quantum of flux, in CGS units, is $\Phi_{0}=2.07 \times 10^{-7} \mathrm{G} \mathrm{cm}{ }^{2}$. A mass anisotropy $\varepsilon^{2}=\left(H_{c 2}^{c} / H_{c 2}^{a b}\right)^{2} \approx 0.3$ is then found for $\mathrm{MgB}_{2}$, which could be considered a mild anisotropy when compared to the highly anisotropic high- Tc cuprates [44], like $\mathrm{YBa}_{2} \mathrm{Cu}_{3} \mathrm{O}_{7-\delta}\left(\varepsilon^{2} \approx 0.04\right)$ and $\mathrm{Bi}_{2} \mathrm{Sr}_{2} \mathrm{CaCu}_{2} \mathrm{O}_{8+x}$ $\left(\varepsilon^{2} \approx 10^{-4}\right)$. 


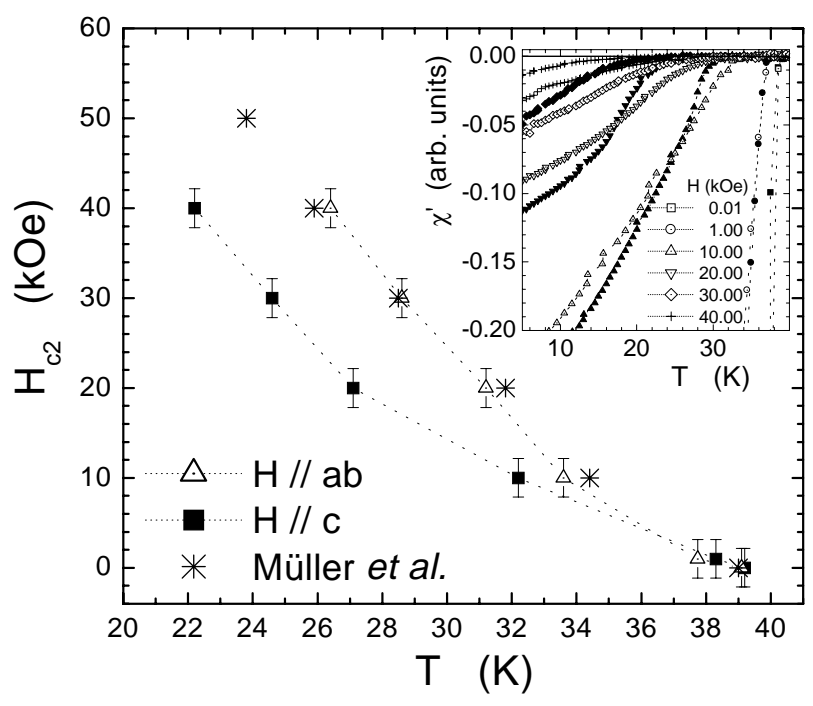

Figure 3. Upper critical field $H_{c 2}$ vs. Temperature phase diagram, for both sample orientations. The stars represent the $H_{c 2}$ vs. $T$ line from Ref. [43]. The inset shows the real component $\chi$ ' of the ac susceptibility vs. temperature, measured at several DC fields for both orientations. Open symbols are for the $H / / a b$ curves and solid symbols for $H / / c$.

In order to study the $H_{c 2}$ angular dependence, under axial applied fields, a special sample rotator was built [41] with all parts machined from a low magnetic teflon rod. Magnetization measurements were performed with a SQUID magnetometer (model MPMS-5, made by Quantum Design). Fig. 4 shows the magnetic dependence of the magnetization in $T=25 \mathrm{~K}$, for a few representative angles, $\theta$, between the sample $c$ axis and the magnetic field direction. The ZFC (Zero Field Cooling) measurements shown in the main frame look noisy possibly due to the effect of intense vortex creep [6], combined with a complex regime of flux penetration in the granular sample of aligned crystallites. Thus, the occurrence of random weak links and the varied coupling between grains contribute also to produce a fluctuating behavior in the sample overall response. However, in all cases it was possible to define $H_{c 2}(\theta)$, at the crossing point between the horizontal baseline and the straight line drawn across the experimental points in the region near the onset of transition. This linear behavior of the magnetization close to the onset is indeed expected from the G-L theory [22]. A constant paramagnetic background was subtracted from all sets of data. In fact one of the reasons for measuring at $\mathrm{T}=$ $25 \mathrm{~K}$ is because at this temperature $H_{c 2}(\theta)$ ranges between 28 - $36 \mathrm{kOe}$, where the paramagnetic background is already saturated [23].

Figure 5 displays $H_{c 2}(\theta)$ for $\theta$ between - $20 \mathrm{deg}$ and $120 \mathrm{deg}$. The vertical error bars were estimated to be around \pm 1 kOe while the horizontal error bars, of \pm 2.5 deg, almost coincide with the symbol size. The solid line going through the experimental points represents a good fit of the angular dependence, predicted by the $3 \mathrm{D}$ anisotropic G-L theory to be $[22,39]$

$$
H_{c 2}(\theta)=H_{c 2}^{c}\left[\cos ^{2}(\theta)+\varepsilon^{2} \sin ^{2}(\theta)\right]^{-1 / 2},
$$

where $\varepsilon^{2}$, is the mass anisotropy ratio. The best fitting is obtained with $\varepsilon^{2} \approx 0.39$, implying that $H_{c 2}^{a b} / H_{c 2}^{c} \approx$ 1.62 , which is close to the value 1.7 anticipated by $\mathrm{AC}$ susceptibility measurements done for the two extreme $\theta$ positions, at 0 and 90 degrees (see Fig. 3). In Fig. 5 we see also five data points $(\theta=0,25,65,85,90 \mathrm{deg})$ marked with stars, which were obtained at the onset of transition of the real part of the complex susceptibility, measured with an excitation field of amplitude $1 \mathrm{Oe}$ and frequency $5 \mathrm{kHz}$.

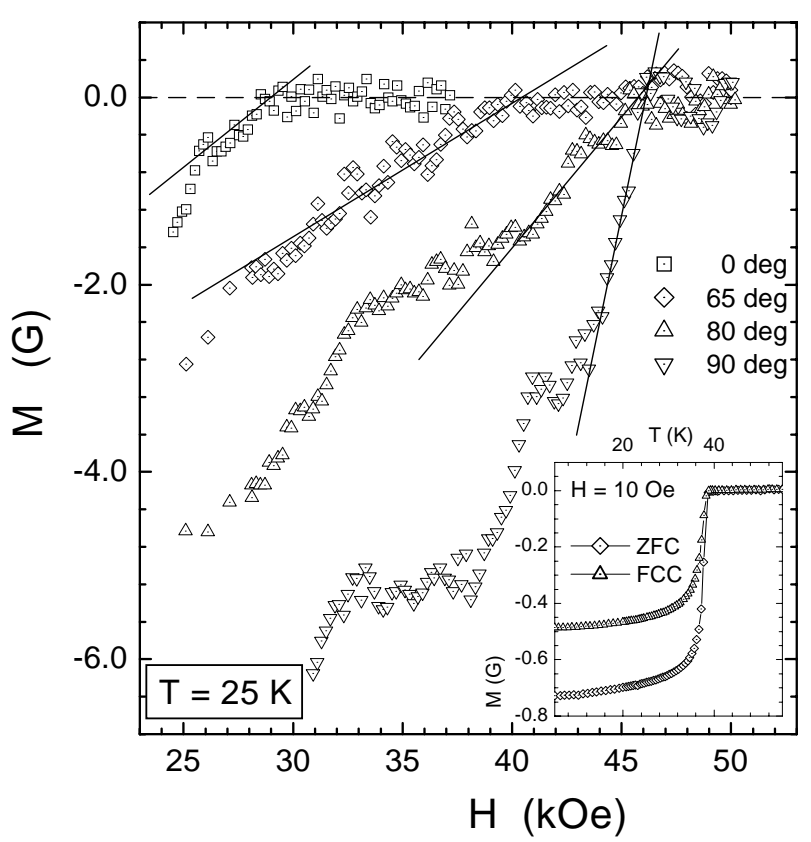

Figure 4. Zero Field Cooling magnetization measurements as a function of the applied field, for $\theta=0,65,80,90$ degrees. The bulk nucleation field $H_{c 2}(\theta)$ is defined at the crossing of the auxiliary straight lines and the horizontal baseline $(M=0)$. The inset shows ZFC and FCC magnetization measurements as a function of temperature for $H=$ 10 Oe, giving $T_{c} \approx 39 \mathrm{~K}$.

The ratio $H_{c 2}^{a b} / H_{c 2}^{c} \approx 1.62$ reminds the relationship predicted for the surface nucleation field [45] $H_{c 3} \approx$ $1.7 H_{c 2}$. However, this is clearly not the case for the presented data, as one can see from the dash-dotted curve in Fig. 5, which is a plot of the expected angular dependence of the surface nucleation field for thick samples, given by [46]:

$$
\left[\frac{H(\theta)}{H_{c 3}} \sin \theta\right]^{2}[1+\cot \theta(1-\cos \theta)]+\frac{H(\theta)}{H_{c 2}} \cos \theta=1,
$$


where $H_{c 3}=H\left(\theta=90^{\circ}\right)$ and $H_{c 2}=H\left(\theta=0^{\circ}\right)$. The dashed curve in between represents the well-known Thinkham's formula[22, 47]

$$
\left[\frac{H(\theta)}{H_{c}^{a b}} \sin \theta\right]^{2}+\left|\frac{H(\theta)}{H_{c}^{c}} \cos \theta\right|=1,
$$

which is valid for the surface nucleation field in very thin films. From both plots one sees that a characteristic feature of the surface nucleation field is a cusplike curve shape near $\theta=90$ degrees. This behavior contrasts with the sinusoidal shape followed by the $\mathrm{MgB}_{2}$ data displayed in Fig. 5. Thus, a strong support is given to interpret the observed upper critical field as a genuine bulk nucleation field.

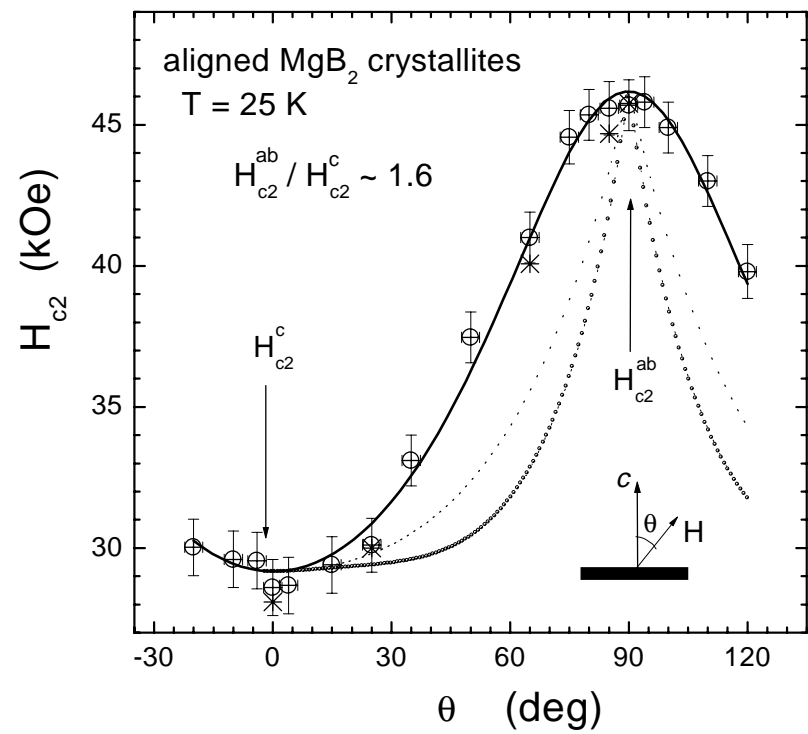

Figure 5. Bulk nucleation field (or upper critical field), $H_{c 2}$, as a function of the angle, $\theta$, between the sample $c$ axis and the magnetic field direction. Plots of the expected angular dependence for the surface nucleation field, $H_{c 3}$, in thick samples (dash-dotted curve) and very thin films (dashed curve) are also shown. The stars at $\theta=0,25,65,85,90$ degrees represent $H_{c 2}(\theta)$ obtained at the onset of transition of the real part of $a c$ susceptibility measurements.

Several other studies have already confirmed the $H_{c 2}$ anisotropy in $\mathrm{MgB}_{2}$. Measurements of resistive transitions on three $c$-axis oriented thin films [48] showed anisotropy ratios $\left(H_{c 2}^{a b} / H_{c 2}^{c}\right)$ of 1.8, 1.9 and 2.0, the ratio increasing with higher resistivity, correlated with a more impure or alloyed sample. Resistivity measurements done on single crystals, having the major linear size around $0.5 \mathrm{~mm}[32,33]$, showed anisotropy ratios close to 2.6. In Ref.[33] the authors have also shown anisotropic $H_{c 1}$ lines pointing to $H_{c 1}^{a b} / H_{c 1}^{c} \approx 1.4$, where $H_{c 1}$ is the lower critical field. However, one should be aware about the large uncertainty usually associated with $H_{c 1}$ evaluations, mainly due to two factors: the very slow departure from linearity, when leaving the Meissner regime, and the strong dependence on demagnetizing fields. Measurements of resistive transitions on very clean epitaxial thin films [26], gave a relatively smaller anisotropy ratio around 1.3 in a large temperature region between 2 - $32 \mathrm{~K}$. A much smaller anisotropy ratio value around 1.1 was also reported on a partially textured, hot deformed, bulk material [49].

More recently the effect of temperature on $H_{c 2}$ anisotropy has been reported by several groups. Measurements of torque magnetometry in $\mathrm{MgB}_{2}$ single crystals have shown a variation from $H_{c 2}^{a b} / H_{c 2}^{c} \approx 2.8$ at 35 $\mathrm{K}$ to $\sim 6$ at $15 \mathrm{~K}$ [50]. Resistive transitions measured in single crystals [51], as well as in c-oriented thin films [52] have also shown the same trend, although in the first case $H_{c 2}^{a b} / H_{c 2}^{c}$ varied between 2.2 and 3.0 when $\mathrm{T}$ decreased from $39 \mathrm{~K}$ to $30 \mathrm{~K}$, while for the second case it varied between 1.5 and 2.0 when $\mathrm{T}$ decreased from $33 \mathrm{~K}$ to $20 \mathrm{~K}$. Contrasting these experimental results a theory based on a two-gap model predicts that $H_{c 2}^{a b} / H_{c 2}^{c}$ should increase from 1.5 do 2.4 , when $\mathrm{T}$ increases from $\sim 18 \mathrm{~K}$ to $\sim 39 \mathrm{~K}[53]$.

The relatively large scattering of values for the $H_{c 2}$ anisotropy ratio, going from 1.3 to approximately 6 , could possibly be ascribed to at least three factors. One is the sample purity, since it affects directly the energy gap anisotropy at the microscopic level [39, 40]. The second is the experimental criterion used to define $H_{c 2}$ or $T_{c 2}$, such that a reliable bulk transition point is actually guaranteed. The third factor is a possible temperature dependence of the anisotropy ratio that could be originated from a temperature dependent gap anisotropy [20, 54]. Therefore, results obtained with samples of different purity levels and measured at different temperatures should not be directly compared without a detailed analysis.

The macroscopic $H_{c 2}$ anisotropy can be caused by an anisotropic gap energy or by an anisotropic Fermi surface, as well as by a combination of both effects. Assuming an isotropic gap, one gets $\xi_{a b} / \xi_{c}=V_{F}^{a b} / V_{F}^{c}$, since [22] $\xi \propto V_{F} / \Delta_{0}$. Therefore, the data for aligned $\mathrm{MgB}_{2}$ crystallites [23, 41] implies $V_{F}^{a b} \approx 1.6 V_{F}^{c}$, for the Fermi velocities within the $a b$ plane and along the $c$ direction. However, several experimental $[15,17,18]$ and theoretical $[11,12,20]$ works have suggested an anisotropic gap energy for $\mathrm{MgB}_{2}$. In particular, two works $[18,20]$ based on the analysis of spectroscopic and thermodynamic data propose an anisotropic s-wave pairing symmetry, such that the minimum gap value, $\Delta_{0} \approx 1.2 k T_{c}$, occurs within the $a b$ plane. Using this result and assuming an isotropic Fermi surface the expected $H_{c 2}$ anisotropy would be $H_{c 2}^{a b} / H_{c 2}^{c} \approx 0.8$. This conflicts with all experimental results that show clearly $H_{c 2}^{a b}>H_{c 2}^{c}$. However, by allowing a Fermi surface anisotropy in their model, Haas and Maki have found that [54] $V_{F}^{a b} \approx 2.5 V_{F}^{c}$ in order to match the ratio $H_{c 2}^{a b} / H_{c 2}^{c} \approx 1.6$, at $T=25 \mathrm{~K}$. Therefore, it seems that the two fundamental sources of microscopic anisotropy is affecting the $H_{c 2}$ anisotropy of $\mathrm{MgB}_{2}$ in opposite ways. As a consequence of combining both effects to explain the $H_{c 2}$ anisotropy, the Fermi velocity anisotropy becomes about $60 \%$ higher when com- 
pared with the isotropic gap hypothesis. Interestingly, a calculation based on a two-band model has also found [55] $V_{F}^{a b} \approx 2.5 V_{F}^{c}$, while a much smaller value of $V_{F}^{a b} \approx 1.03 V_{F}^{c}$ was found in a band structure calculation using a general potential method [11].

\section{Other anisotropic properties}

Another anisotropic property already assessed in the superconducting state of $\mathrm{MgB}_{2}$ is the field penetration depth $\lambda[56]$. Using a radio frequency technique $\lambda(T)$ was measured in polycrystalline samples and its anisotropic values were evaluated, from a theoretical analysis, to be around $\lambda_{a b} \approx 1200 \AA$ and $\lambda_{c} \approx 2450 \AA$.

Besides the strongly anisotropic crystalline structure of $\mathrm{MgB}_{2}$, three other normal state anisotropic properties have been identified, the magnetoresistance, the compressibility and the thermal expansion. The magnetoresistance measured at $T=45 \mathrm{~K}$, in epitaxial thin films, was found [26] to increase monotonically up to $8 \%$ for $H / / c$ and up to $13 \%$ for $H / / a b$, when $\mathrm{H}$ goes up to $60 \mathrm{~T}$. Concerning the compressibility studies, similar results were obtained by two groups, one [24] using synchrotron $\mathrm{X}$-ray diffraction and applying up to $6 \mathrm{GPa}$ of pressure on the sample and the other [25] using neutron powder diffraction and applying up to $0.6 \mathrm{GPa}$ of pressure. They followed the relative lattice parameters variation as a function of applied pressure, at room temperature, and found that the compression along the $c$ axis is about $64 \%$ larger than along the $a$ axis. Ref.[25] reports also that the thermal expansion (between $11 \mathrm{~K}-297 \mathrm{~K}$ ) along the $c$ axis is about twice that along the $a$ axis.

Although no experimental results on $J_{c}$ anisotropy was reported yet, it could be anticipated that the inplane critical current density values are expected to be at least about $60 \%$ higher than the values along the $c$ axis direction $(H / / a b)$. This result is expected because $J_{c}$ is proportional to $\xi^{2}$, therefore [44] $J_{c}(H / / c) / J_{c}(H / / a b) \approx \xi_{a b} / \xi_{c} \approx H_{c 2}^{a b} / H_{c 2}^{c}$. This means that in order to optimise $J_{c}$ in wires or other polycrystalline components some texturization technique will be useful.

\section{Conclusion}

Starting at the beginning of 2001 an intense activity has been devoted to the study of the binary compound $\mathrm{MgB}_{2}$, since it was found to be a superconductor for temperatures below $39 \mathrm{~K}$. The anisotropic crystalline structure of $\mathrm{MgB}_{2}$ consists of triangular layers of Magnesium atoms sandwiched between hexagonal layers of Boron atoms. Therefore, it should indeed be expected some anisotropy in its physical and chemical properties.

This paper presented a brief review on the already reported anisotropic properties of $\mathrm{MgB}_{2}$, in the superconducting state (e.g. upper critical field $H_{c 2}$, field penetration depth $\lambda$, coherence length $\xi$, and energy gap $\Delta$ ) as well as in the normal state (e.g., magnetoresistance, compressibility, thermal expansion). So far, the reported results have been carried out using aligned crystallites, c-axis oriented thin films and submillimiter crystals. However, good-sized single crystals with linear dimensions above $1 \mathrm{~mm}$ are still highly desirable. This will open the possibility of new advances, making easier to probe directly other expected anisotropic properties, like the critical current density and the normal state resistivity, among others. Since impurity scattering affects directly the gap anisotropy it will be also of interest to know the effects of crystal purity on the anisotropy factors. Most possibly this could be one of the main reasons for the scattered values observed in the $H_{c 2}$ anisotropy ratio, ranging between 1.3 and 6.

\section{Acknowledgements}

I would like to thank my close collaborators C. A. Cardoso, R. A. Ribeiro, M. A. Avila and A. A. Coelho, for their help in the anisotropy studies on the $\mathrm{MgB}_{2} \mathrm{su}-$ perconductor. I acknowledge the financial support from the Brazilian Science Agencies FAPESP and CNPq.

\section{References}

[1] J. Nagamatsu, N. Nakagawa, T. Muranaka, Y. Zenitani, and J. Akimitsu, Nature 410, 63 (2001).

[2] D.C. Larbalestier, L.D. Cooley, M.O. Rikel, A.A. Polyanskii, J. Jiang, S. Patnaik, X.Y. Cai, D.M. Feldmann, A. Gurevich, A.A. Squitieri, M.T. Naus,C.B. Eom, E.E. Hellstrom, R.J. Cava, K.A. Regan, N. Rogado, M.A. Hayward, T. He, J.S. Slusky, P. Khalifah, K. Inumaru, and M. Haas, Nature 410, 186 (2001).

[3] P.C. Canfield, D.K. Finnemore, S.L. Bud'ko, J.E. Ostenson, G. Lapertot, C.E. Cunningham, and C. Petrovic, Phys. Rev. Lett. 86, 2423 (2001).

[4] C.B. Eom, M.K. Lee, J.H. Choi, L.J. Belenky, X. Song, L.D. Cooley, M.T. Naus, S. Patnaik, J. Jiang, M. Rikel, A.Polyanskii, A. Gurevich, X.Y. Cai, S.D. Bu, S.E. Babcock, E.E. Hellstrom, D.C. Larbalestier, N. Rogado, K.A. Regan, M.A. Hayward, T. He, J.S. Slusky, K. Inumaru, M.K. Haas, and R.J. Cava, Nature 411, 558 (2001).

[5] S. Jin, H. Mavoori, C. Bower, and R.B. van Dover, Nature 411, 563 (2001).

[6] Y. Bugoslavski, G.K. Perkins, X. Qi, L.F. Cohen, and A.D. Caplin, Nature 410, 563 (2001).

[7] Y. Bugoslavsky, L.F. Cohen, G.K. Perkins, M. Polichetti, T.J. Tate, R. Gwilliam, A.D. Caplin, Nature 411, 561 (2001).

[8] S.L. Bud'ko, G. Lapertot, C. Petrovic, C.E. Cunningham, N. Anderson, and P.C. Canfield, Phys. Rev. Lett. 86, 1877 (2001).

[9] D.G. Hinks, H. Claus, and J. D. Jorgensen, Nature 411, 457 (2001). 
[10] G. Karapetrov, M. Iavarone, W. K. Kwok, G. W. Crabtree, and D. G. Hinks, Phys. Rev. Lett. 86, 4374 (2001).

[11] J. Kortus, I. I. Mazin, K. D. Belashchenko, V. P. Antropov, and L. L. Boyer, Phys. Rev. Lett. 86, 4656 (2001).

[12] J. M An and W. E. Pickett, Phys. Rev. Lett. 86, 4366 (2001).

[13] N. I. Medvedeva, A. L. Ivanovskii, J. E. Medvedeva, and A. J. Freeman, Phys. Rev. B 64, 20502, (2001).

[14] R. Osborn, E. A. Goremychkin, A. I. Kolesnikov, and D. G. Hinks, Phys. Rev. Lett. 87, 017005 (2001).

[15] Y. Wang, T. Plackowski, and A. Junod, Physica C 355, 179 (2001).

[16] T. Yildirim, O. Gü;seren, J.W. Lynn, C.M. Brown, T.J. Udovic, Q. Huang, N. Rogado, K.A. Regan, M.A. Hayward, J.S. Slusky, T. He, M.K. Haas, P. Khalifah, K. Inumaru, and R.J. Cava, Phys. Rev. Lett. 87, 037001 (2001).

[17] F. Bouquet, R. A. Fisher, N. E. Philips, D. G. Hinks, and J. D. Jorgensen, Phys. Rev. Lett. 87, 047001 (2001).

[18] P. Seneor, C.-T. Chen, N.-C. Yeh, R.P. Vasquez, L.D. Bell, C.U. Jung, Min-Seok Park, Heon-Jung Kim, W.N. Kang, and Sung-Ik Lee, Phys. Rev. B 65, 012505 (2001).

[19] A.Y. Liu, I.I. Mazin, and J. Kortus, Phys. Rev. Lett. 87, 087005 (2001)

[20] S. Haas and K. Maki, Phys. Rev. B 65, 020502 (2001); A. I. Posazhennikova et al., Europhysics Letters (in print, 2002).

[21] J.E. Hirsch, Phys. Lett. A 282, 392 (2001).

[22] M. Tinkham, Introduction to Superconductivity (McGraw-Hill, New York, 1996).

[23] O.F. de Lima, R.A. Ribeiro, M.A. Avila, C.A. Cardoso, and A.A. Coelho, Phys. Rev. Lett. 86, 5974 (2001).

[24] K. Prassides, Y. Iwasa, T. Ito, Phys. Rev. B 64, 012509 (2001).

[25] J.D. Jorgensen, D.G. Hinks, and S. Short, Phys. Rev. B 63, 224522 (2001).

[26] M.H. Jung, M. Jaime, A.H. Lacerda, G.S. Boebinger, W.N. Kang, H.J. Kim, E.M. Choi, and Sung-Ik Lee, Chem. Phys. Lett. 343, 447 (2001).

[27] C. Buzea and T. Yamashita, Supercond. Sci. Technol. 14, R115 (2001).

[28] see e.g.: A. A. Nayeb-Hashemi and J. B. Clark (ed.), Phase Diagrams of Binary Magnesium Alloys (ASM International, Metals Park, Ohio, 1988).

[29] V. Russel, R. Hirst, F.A. Kanda, and A.J. King, Acta Crystallogr. 6, 870 (1953)

[30] M.E. Jones and R.E. Marsh, J. Am. Chem. Soc. 76 1434 (1954).

[31] MM. R. Naslaim, A. Guette, and M. Barret, J. Solid State Chem. 8, 68 (1973).

[32] S. Lee, H. Mori, T. Masui, Y. Eltsev, A. Yamamoto, and S. Tajima, J. Phys. Soc. Jpn. 70, 2255 (2001).

[33] M. Xu, H. Kitazawa, Y. Takano, J. Ye, K. Nishida, H. Abe, A. Matsushita, N. Tsujii, and G. Kido, Appl. Phys. Lett. 79, 2779 (2001).
[34] W.N. Kang, H.-Jin Kim, E.-Mi Choi, C.U. Jung, and S.-Ik Lee, Science 292, 1521 (2001).

[35] H.Y. Zhai, H.M. Christen, L. Zhang, A. Paranthaman, C. Cantoni, B.C. Sales, P.H. Fleming, D.K. Christen, and D.H. Lowndes, J. Mater. Res. 16, 2759 (2001).

[36] G.V. Raynor, The Physical Metallurgy of Magnesium and its Alloys (Pergamon, London, 1959), p. 441.

[37] Y. Moritomo and Sh. Xu, cond-mat/0104568 at $<$ http://xxx.lanl.gov> (2001).

[38] T.B. Massalski (editor), Binary Alloy Phase Diagrams (ASM International, Metals Park, OH, 1990), $2^{\text {nd }}$ ed.

[39] D.R. Tilley, Proc. Phys. Soc. London 86, 289 (1965).

[40] H.W. Weber (ed.), Anisotropy Effects in Superconductors (Plenum Press, New York, 1977).

[41] O.F. de Lima, C.A. Cardoso, R.A. Ribeiro, M.A. Avila, and A.A. Coelho, Phys. Rev. B 64, 144502 (2001).

[42] D.K. Finnemore, J.E. Ostenson, S.L. Bud'ko, G. Lapertot, and P.C. Canfield, Phys. Rev. Lett. 86, 2420 (2001).

[43] K.-H. Müller, G. Fuchs, A. Handstein, K. Nenkov, V.N. Narozhnyi, and D. Eckert, J. Alloy Compd. 322, L10 (2001).

[44] G. Blatter, M.V. Feigel'man, V.B. Geshkenbein, A.I. Larkin, and V.M. Vinokur, Rev. Mod. Phys. 66, 1125 (1994).

[45] D. Saint-James and P.G. de Gennes, Physics Letters 7, 306 (1963).

[46] K. Yamafuji, E. Kusayanagi, and F. Irie, Physics Letters 21, 11 (1966).

[47] M. Tinkham, Physics Letters 9, 217 (1964).

[48] S. Patnaik, L.D. Cooley, A. Gurevich, A.A. Polyanskii, J. Jing, X.Y. Cai, A.A. Squitieri, M.T. Naus, M.K. Lee, J.H. Choi, L. Belensky, S.D. Bu, J. Letteri, X. Song, D.G. Schlom, S.E. Babcock, C.B. Eom, E.E. Hellstrom, and D.C. Larbalestier, Supercond. Sci. Technol. 14, 315 (2001).

[49] A. Handstein, D. Hinz, G. Fuchs, K.H. Muller, K. Nenkov, O. Gutfleisch, V.N. Narozhnyi, and L. Schultz, J. Alloy Compd. 329, 285 (2001).

[50] M. Angst, R. Puzniak, A. Wisniewski, J. Jun, S.M. Kazakov, J. Karpinski, J. Roos, and H. Keller, Phys. Rev. Lett. 88, 167004 (2002).

[51] Yu. Eltsev, S. Lee, K. Nakao, N. Chikumoto, S. Tajima, N. Koshizuka, and M. Murakami, Phys. Rev. B 65, 140501 (2002).

[52] C. Ferdeghini, V. Braccini, M.R. Cimberle, D. Marré, P. Manfrinetti, V. Ferrando, M. Putti, and A. Palenzona, cond-mat/0203246 at <http://xxx.lanl.gov> (2002).

[53] V.G. Kogan, cond-mat/0204038 at $<$ http://xxx.lanl.gov> (2002).

[54] S. Haas, private communications (2001).

[55] S. V. Shulga, S. -L. Drechsler, H. Eschrig, H. Rosner, and W. E. Pickett, cond-mat/0103154 at <http://xxx.lanl.gov> (2001).

[56] F. Manzano and A. Carrington, Phys. Rev. Lett. 88, $047002(2002)$ 